\title{
Varizenchirurgie bei Adipositas
}

\author{
G. Bruning'; M. Donath' ${ }^{2}$; J. K. Buhr' \\ ${ }^{1}$ Krankenhaus Tabea GmbH \& Co. KG, Hamburg; ${ }^{2}$ Allergie- und Hautpraxis, Brugg/Schweiz
}

\section{Schlüsselwörter}

Chronisch venöse Insuffizienz, Adipositas, Varizenchirurgie, Crossenrevision, Endovenöse Therapie

\section{Zusammenfassung}

Die operative Sanierung der Varikosis stellt bei korrektem intraoperativen Management auch bei adipösen Patienten eine hervorragende Therapieoption dar. Bei der operativen Sanierung der Vena saphena parva und der Seitenastvarikosis ist das Körpergewicht vernachlässigbar, die operative Therapie der Vena saphena magna muss jedoch gewichtsadaptiert geplant und angepasst werden: Der operative Zugang sollte oberhalb der Leistenbeuge und ausreichend groß gewählt werden, die Stumpfligatur mit nicht resorbierbarem Nahtmaterial doppelt ligiert und das Stumpfendothel koaguliert werden. Crossenrezidive stellen insbesondere bei adipösen Patienten sowohl nach operativer Sanierung der Varikosis, als auch nach endovenösen Therapieverfahren eine operative Herausforderung dar. Die Komplikationsrate bei operativer Sanierung ist sehr niedrig. Die Infektionsrate liegt bei adipösen Patienten nur marginal oberhalb derer von Patienten mit einem

Korrespondenzadresse

Dr. med. Guido Bruning

Chefarzt - Krankenhaus Tabea GmbH \& Co.KG

Kösterbergstr. 32, 22587 Hamburg

E-Mail: gbruning@tabea-krankenhaus.de
$\mathrm{BMI}<30$. Eine Risikoeinschätzung von endovenösen Therapien bei adipösen Patienten steht aktuell aus. Es besteht allerdings eine höhere Rezidivneigung bei größerlumigen $\mathrm{Ge}$ fäßen.

\section{Keywords}

Chronic venous insufficiency, obesity, varicose vein surgery, recurrent varicose veins, endovenous treatment

\section{Summary}

Varicose vein surgery outlines an excellent treatment option among overweight and obese patients. The impact of obesity varies pending on the planed clinical finding. Despite obesity vena saphena parva surgery as well as surgery of side branch varicose veins are fairly unproblematic. However, varicose vein surgery of vena saphena magna has to be planned and adapted to overweight. Apart from very few exceptions (e.g. leg ulcer) BMI 40 represents the maximum BMI for varicose vein surgery at our hospital. Starting from BMI $\geq 30$ we add general anaesthesia to local tumescent anaesthesia. When operating obese pa-

Varicose vein surgery and obesity

Phlebologie 2018; 47: 71-74

https://doi.org/10.12687/phleb2415-2-2018

Eingereicht: 31. Januar 2018

Angenommen: 18. Februar 2018

English version available at: www.phlebologieonline.de tients surgical access should be chosen above the groin in order to reduce the number of wound infections. Furthermore, operational access should be chosen great enough to ensure a sufficient preparation considering depth and width. In case of bleeding a great operational access is required to achieve successful haemostasis. Non-absorbable sutures should be used for ligation of the vessel and endothelial tissue should be coagulated at the end in order to reduce the number of recurrent veins. Both, after varicose vein surgery and after endovenous therapy, recurrent varicose veins represent a great challenge for the physician in charge. A modified surgical access named after Junod proves to be successful. Overall the rate of complications during and after varicose vein surgery is low. Nerve injuries, deep vein thrombosis, bleeding, wound infections and postoperative seroma outline the most common complications of varicose vein surgery. Most complications are not effected by obesity. Surprisingly the number of postoperative seroma at BMI $>30$ decreases, nevertheless it appears more frequent after recurrent varicose vein surgery. There are no data available representing lower treatment risks of endovenous therapy in comparison to varicose vein surgery of obese patients. However, greater veins diameter (especially vena saphena magna) decreases the rate of success and increase the recurrent varicose veins significantly after endovenous therapy. Therefore, we prefer varicose vein surgery from veins diameter $>10 \mathrm{~mm}$.

\section{Einleitung}

Die chronisch venöse Insuffizienz ist eine sehr häufige Krankheit in der Bundesrepublik Deutschland. Ca. 10 \% aller Deutschen haben einen therapiebedürftigen venösen Befund und ca. $1 \%$ leiden unter einem venösen Ulcus cruris (1).
Die Inzidenz der Adipositas hat in den letzten Jahren deutlich zugenommen (2). Dies führt auch zu einem steigenden Anteil von adipösen Patienten mit chronisch venöser Insuffizienz $(3,4)$. Die operative Sanierung der Varikosis bei Adipositas stellt eine besondere Herausforderung dar.

\section{Indikationsstellung zur operativen Therapie bei Adipositas}

Die Gewichtung der Adipositas ist bei der operativen Sanierung der chronisch venösen Insuffizienz befundabhängig. Eine operative Sanierung der Varikosis bei Adiposi- 


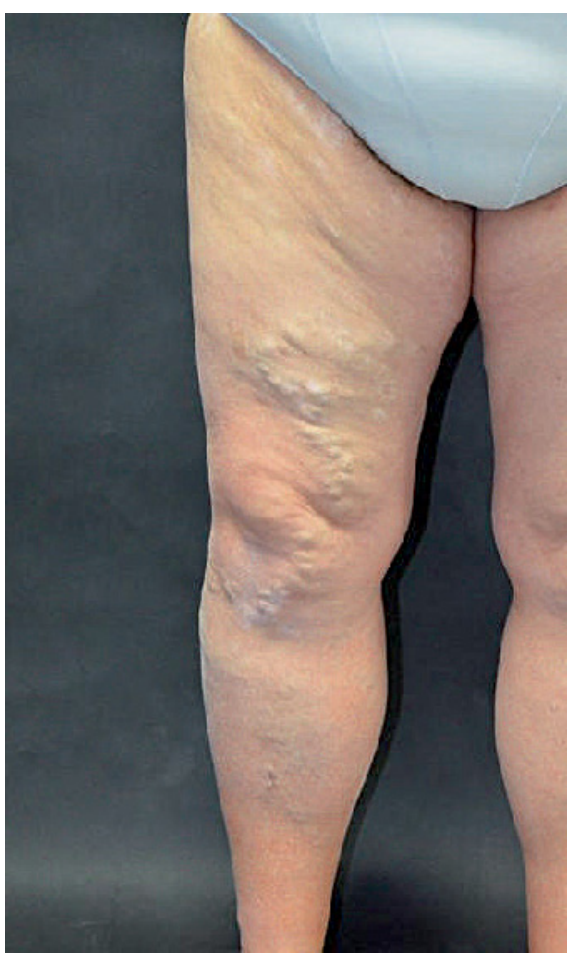

Abb. 1 Crossenrezidivvarikosis bei Adipositas

tas sollte nur bei absolut sicherer Behandlugsbedürftigkeit erfolgen - d.h. bei einer symptomatischen Varikosis. Die positive Korrelation zwischen Adipositas und dem Fortschreiten einer bestehenden chronisch venösen Insuffizienz muss berücksichtig werden (3).

Bei der operativen Sanierung der Vena saphena parva durch popliteale Crossektomie und Stripping sowie Seitenastexhairese spielt die Adipositas nur eine untergeordnete Rolle, da der operative Zugang durch die bestehende Adipositas in der Regel nicht erheblich eingeschränkt wird.

Diese Operation ist in der Regel in Tumeszenzlokalanästhesie ohne Allgemein-

Tab. 1 Zusammensetzung der bei uns verwendeten 0,069\%igen Tumeszenzlokalanästhesie (TLA)

\begin{tabular}{l|r|r|}
\hline & \multicolumn{1}{|c|}{ Liter } & \multicolumn{1}{c}{ Liter } \\
\hline NaCl-Lösung 0,9\% & $3000 \mathrm{ml}$ & $5000 \mathrm{ml}$ \\
\hline $\begin{array}{l}\text { Prilocain 1\% (Xylonest) } \\
\text { Natriumhydrogencarbonat }\end{array}$ & $225 \mathrm{ml}$ & $375 \mathrm{ml}$ \\
\hline $\begin{array}{l}8,4 \% \\
\begin{array}{l}\text { Epinephrin (Suprarenin) } \\
\text { 1:1000 }\end{array}\end{array}$ & $30 \mathrm{ml}$ \\
\hline
\end{tabular}

anästhesie komplikationslos durchzuführen. Die maximale Belastbarkeit des OPTisches ist hier ein limitierender Faktor.

Bei der operativen Sanierung der Vena saphena magna stellt die Adipositas ein nicht unbedeutendes Operationshindernis dar. Die Adipositas erschwert die Erreichbarkeit der Crossenmündung in der Tiefe erheblich.

$\mathrm{Ab}$ einem BMI 30 planen wir alle operativen Sanierungen zusätzlich zur Tumeszenzlokalanästhesie in Allgemeinnarkose, weil die alleinige Tumeszenzlokalanästhesie in der Tiefe des Operationsgebietes häufig nicht ausreicht.

Als Obergrenze für die operative Sanierung der Vena saphena magna haben wir in unserem Hause einen BMI von 40 festgelegt. Dieser wird nur im Einzelfall z.B. bei einem floridem Ulcus cruris überschritten.

\section{Operationstechnik bei chronisch venöser Insuffizienz und Adipositas}

Der operative Zugang sollte bei Adipositas oberhalb der Leistenbeuge erfolgen.

Durch diesen Zugangsweg wird verhindert, dass die Inzision in der feuchten Leistenbeuge verschwindet und so das vermehrte Auftreten von postoperativen Wundinfektionen vermieden.

Außerdem sollte der operative Zugangsweg groß genug gewählt werden, sodass eine ausreichende Präparation in der Tiefe und der Breite gewährleistet werden kann und bei dem eventuellen Auftreten von Blutungskomplikationen ein sicherer $\mathrm{Zu}$ gangsweg besteht.

Wichtig ist, bei der Radikalität in der Tiefe nicht zurückhaltend zu operieren, um eine Rezidivsituation (5), die eine deutliche größere operative Herausforderung ist, zu vermeiden ( $\triangleright$ Abb. 1$)$.

Die Stumpfligatur sollte mit nicht resorbierbarem, nicht verrottendem Nahtmaterial erfolgen, um eine optimale Crossenversorgung mit einer niedrigen Rezidivquote sicherzustellen. Wir führen eine doppelte Ligatur durch, alternativ kann eine Durchstechungsligatur erfolgen. Außerdem sollte das freiliegende Stumpfendothel koaguliert oder übernäht werden, um einer Neoan- giogenese - ein häufiger Auslöser der Crosssenrezivivarikosis - vorzubeugen.

Zusätzlich zur Allgemeinnarkose führen wir diese Operationen in Tumeszenzlokalanästhesie in einer 0,069\%igen Prilocain-Lösung ( Tab. 1) durch. Wir limitieren die Maximaldosis auf 20mg/kg KG (6). Dies führt auch bei adipösen Patienten zu weniger ausgeprägten Hämatomen und einer postoperativ geringeren Schmerzsymptomatik, sodass wir in der Regel mit einer oralen peripheren Schmerzmedikation auskommen.

\section{Operative Sanierung der Crossenrezidivvarikosis bei adipösen Patienten}

Ca. 8-10\% der Patienten entwickeln 5 Jahre postoperativ ein klinisch relevantes Rezidiv. (4) Diese Quote kann mit moderneren Operationstechniken und bei Verwendung von nicht-resorbierbarem Nahtmaterial auf unter 5\% gesenkt werden. (7) Die Crossenrezidivvarikosis stellt eine besondere operative Herausforderung dar, da sich im Operationsbereich die Vernarbungen der Voroperation befinden.

In zunehmendem Maße zeigen sich auch Crossenrezidive nach vorausgegangenen endovenösen Therapieverfahren. Auch diese gehen in der Regel mit einer vermehrten Narbenbildung in der Crosse einher.

Bei der operativen Sanierung der Crossenrezidivvarikosis hat sich der modifizierte Leistenzugang nach Junod bewährt (8).

Hierbei erfolgt über einen inguinalen Querschnitt die longitudinale Präparation der Arteria femoralis, danach die Präparation medial der Vena femoralis communis, um unter der alten Narbenplatte den Stumpf der Vena saphena magna freizupräparieren. ( $\triangleright$ Abb. 2, $\triangleright$ Abb. 3) Häufig ist dieser bei sehr adipösen Patienten unversehrt, da der Voroperateur die tiefen Regionen nicht erreicht hat. Nach doppelter Ligatur des Stumpfes ( $>$ Abb. 4) an der Mündung kann dann das Rezidivbeet aus der Narbenplatte scharf herausgetrennt werden, ohne dass ein erhöhtes Blutungsrisiko besteht. 
Dieses Vorgehen hat sich auch bei Narbenstümpfen nach endoluminaler Laseroder Radiofrequenzablation bewährt.

\section{Komplikationen in der Varizenchirurgie}

Typische Komplikationen nach der operativen Sanierung der Varikosis sind Nervenverletzungen, tiefe Beinvenenthrombosen, Blutungen, Wundinfektionen sowie die Ausbildung eines postoperativen Seroms (9).

Insgesamt ist die Komplikationsrate der operativen Sanierung der Varikosis sehr niedrig. ( Tab. 2) Die Infektionsrate liegt bei der Indikatoroperation, der inguinalen Re-Crossektomie, in unserem Hause bei $1,2 \%$.

Bei der postoperativen Thrombose sind überdurchschnittlich häufig Patienten mit einer Vena-saphena-parva-Exhairese betroffen. Da nur symptomatische postoperative Thrombosen bei uns in die Erhebung mit einfließen, kann die postoperativen Thromboserate lediglich geschätzt werden, sie liegt jedoch deutlich unter 0,5\%.

Bei einer internen Komplikationsanalyse an 1475 Patienten mit 1803 Operationen zeigte sich eine erfreulich geringe postoperative Nervenverletzungs- und Blutungskomplikationsrate. Intraoperativ kam es in $0,4 \%$ der $n=1803$ Operationen, d.h. $7 \mathrm{mal}$ zu starken Blutungen.

Transfusionspflichtige Blutungskomplikationen sind in unserer Patientenklientel in den letzten 14 Jahren bei ca. 30000 durchgeführten Operationen nicht aufgetreten.

Die Nervenverletzungsquote, mit nach 3 Monaten persistierenden Parästhesien, liegt mit 2,99\% im überschaubaren Bereich. Hier zeigen adipöse Patienten im Vergleich zu schlanken Patienten ein deutlich niedrigeres Nervenverletzungsrisiko. Die Adipositas ist also nicht in allen Bereichen mit einer erhöhten Komplikationsrate assoziiert,

Die Infektionsquote liegt bei adipösen Patienten tendenziell etwas über der Infektionsquote der Patienten mit einem BMI 30. Nimmt man die Komplikationen Abszess und Erysipel jedoch zusammen als eine Infektionsgruppe (gemäß CDC Krite-
Abb. 2

Modifizierter Zugang nach Junod: Darstellung der Arteria femoralis, Vena femoralis, Crossenstumpf der Vena saphena magna

Abb. 3

Umfahren des Crossenstumpfes der Vena saphena magna mit dem Mixter

Abb. 4

Abgesetzter Crossenstumpf der Vena saphena magna mit doppelter nicht resorbierbarer, nicht verrottender Ligatur

Tab. 2

Postoperative Komplikationen; die Prozentzahlen beziehen sich auf die jeweilige Gesamtzahl n der operierten Beine, für die vom Patienten der Fragebogen Post-OP ausgefüllt wurde.
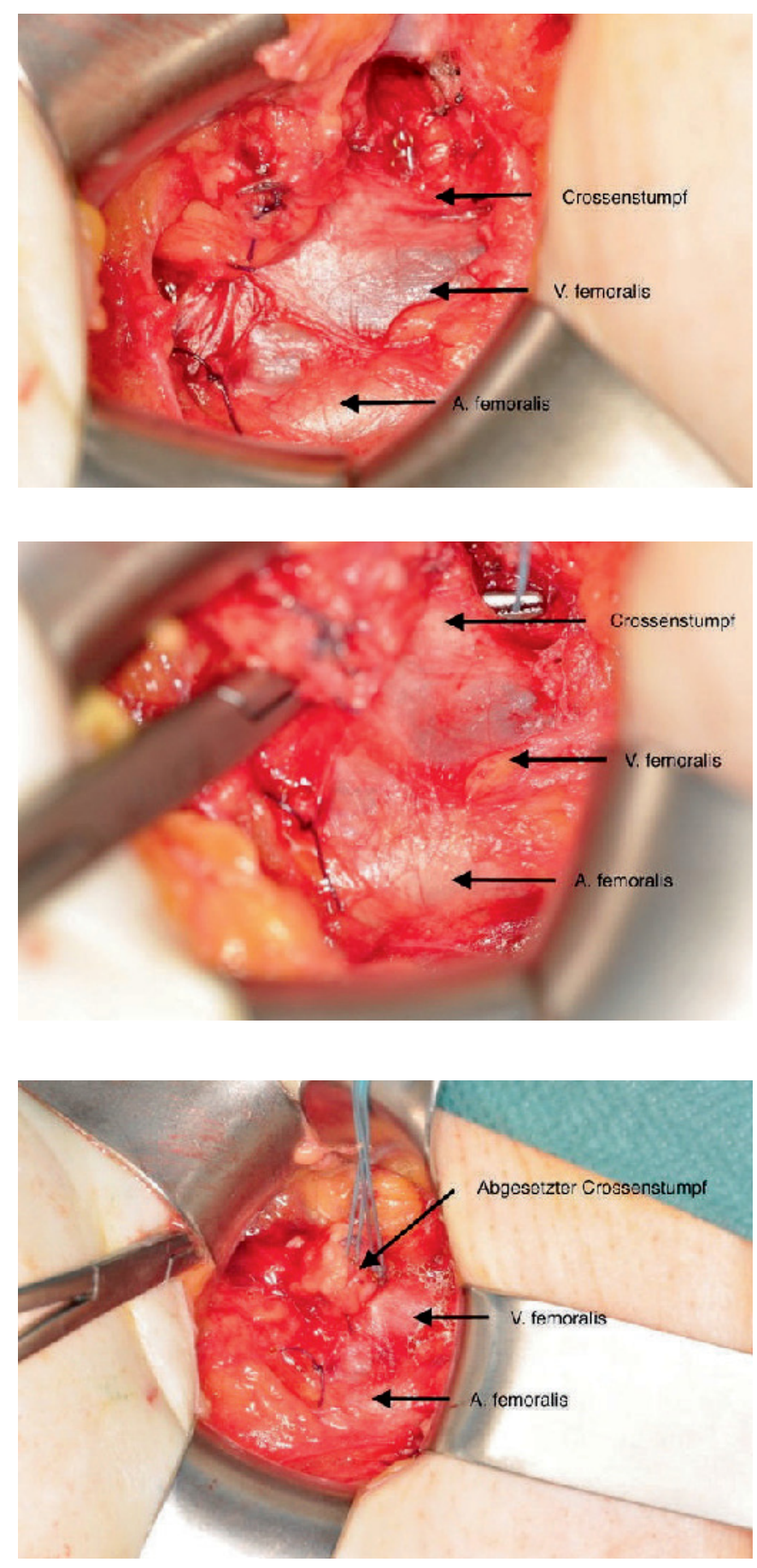

\begin{tabular}{l|r|c|r|c}
\hline & $\begin{array}{l}\text { Gesamt } \\
\mathbf{n}=1641\end{array}$ & $\begin{array}{l}\text { Rezidiv } \\
\mathbf{n}=227\end{array}$ & $\begin{array}{l}\text { BMI } \leq 30 \\
\mathbf{n}=1372\end{array}$ & $\begin{array}{l}\text { BMI > 30 } \\
\mathbf{n}=262\end{array}$ \\
\hline Abszess & $16(1,0 \%)$ & $8(3,5 \%)$ & $10(0,7 \%)$ & $6(2,3 \%)$ \\
\hline Erysipel & $7(0,4 \%)$ & $3(1,3 \%)$ & $5(0,4 \%)$ & $2(0,8 \%)$ \\
\hline Serom & $63(3,8 \%)$ & $19(8,4 \%)$ & $57(4,2 \%)$ & $6(2,3 \%)$ \\
\hline Hämatom & $35(2,1 \%)$ & $7(3,1 \%)$ & $29(2,1 \%)$ & $6(2,3 \%)$ \\
\hline Schwellung & $143(8,7 \%)$ & $32(14,1 \%)$ & $105(7,7 \%)$ & $36(13,7 \%)$ \\
\hline Thrombose & $1(0,1 \%)$ & $0(0 \%)$ & $0(0,0 \%)$ & $1(0,4 \%)$ \\
\hline
\end{tabular}


rien) ergeben sich signifikante Unterschiede zwischen den beiden Gruppen $\mathrm{p}=0,0161<0,05$. Bei den Patienten mit BMI >30 $(n=262)$ bekamen 3,05\% eine Infektion. Dagegen waren es bei den schlanken Patienten mit BMI $\leq 30 \quad(n=1372)$ lediglich 1,02\%."

$\mathrm{Zu}$ unserem eigenen Erstaunen haben Patienten mit einem BMI > 30 ein deutlich niedrigeres Seromrisiko als Patienten mit einem $\mathrm{BMI}<30$. Rezidivoperation sind mit einem deutlich erhöhten Seromrisiko gegenüber der Primäroperation verknüpft. Dies lässt sich auf Grund der vermehrten Beschädigung von Lymphbahnen durch die scharfe Präparation im Narbengewebe und im Bereich der Vena femoralis communis erklären.

\section{Stellenwert der Operation gegenüber endovenösen Therapieverfahren}

Bei endovenösen Therapieverfahren wird häufig ein niedrigeres Behandlungsrisiko gegenüber der klassischen Operation suggeriert. $\mathrm{Zu}$ adipösen Patienten liegen diesbezüglich jedoch keine eindeutigen Daten vor.

Allerdings nimmt die Erfolgsquote der endovenösen Therapieverfahren bei einem größeren Venendurchmesser der Vena saphena magna deutlich ab und die Rezidivhäufigkeit steigt. Nach endovenöser Therapie kommt es bei ca. 10\% der Patienten $\mathrm{zu}$ inguinalen Refluxen gegenüber $<5 \%$ inguinalen Refluxen nach klassischer Crossektomie und Stripping (7).

Dies konnte gerade jetzt in einer neuen Metaanalyse nachgewiesen werden (10).
Die perioperative Morbidität, Mortalität und Lebensqualität unterscheidet sich bei beiden Therapieverfahren nicht signifikant. Schon die klassische Therapie der Varikosis mit Crossektomie und Stripping bringt eine erhebliche Verbesserung der Lebensqualität für die Patienten auch im Langzeitverlauf (4).

Infolgedessen präferieren wir bei einem Venendurchmesser von $>10 \mathrm{~mm}$ im Crossenbereich die klassische operative Sanierung der Varikosis gegenüber einem endovenösen Therapieverfahren. Auch beim Vorliegen der Vena-accessoria-anterior-Insuffizienz vom inguinalen Mündungstyp erscheint das Rezidivrisiko nach endovenösen Therapieverfahren höher, sodass wir auch in diesem Fall die klassische Operation bevorzugen (11). Aufgrund des einfacheren Handlings ist jedoch bei geeigneten Befunden - Venendurchmesser $<10 \mathrm{~mm}$ ) und Kostenübernahme der Krankenkasse ein endovenöses Therapieverfahren zu bevorzugen.

\section{Fazit}

Adipositas stellt in der Varizenchirurgie eine Herausforderung für den Operateur dar. Bei ausreichender Erfahrung und angepasstem operativen Setting sind auch bei Adipositas sehr gute postoperative Ergebnisse bei geringen Komplikationsraten zu erzielen.

\section{Interessenkonflikt}

Nach Angaben der Autoren bestehen keine Interessenkonflikte.

\section{Ethische Richtlinien}

Für das Manuskript wurden keine Studien an Menschen oder Tieren durchgeführt.

\section{Literatur}

1. Rabe E, Pannier-Fischer F, Bromen K, Schuldt K, Stang a, Poncar C, et al. Bonner Venenstudie der Deutschen Gesellschaft für Phlebologie. Phlebologie 2003; 32(1): 1-14.

2. Smith KB, Smith MS. Obesity Statistics 2016; 121-35.

3. Göstl K, Obermayer A, Hirschl M. Pathogenesis of chronic venous insufficiency by obesity. Phlebologie 2009; 108-13.

4. Faubel R, Schäfer I, Augustin M, Bruning G. Langzeitergebnisse und Analysen von Zusammenhängen 5 Jahre nach Varizenstripping. Phlebologie 2010; 263-269.

5. Mann S. Chirurgie der Rezidivvarikose. Chirurgen Magazin 2008.

6. Bruning G, Standl T, Diedrich A, I. Moll. Prilocaine pharmacokinetics and the influence of vitamin $\mathrm{C}$ on methaemoglobin concentrations in tumescent anaesthesia. Phlebologie 2007; 145-150.

7. Gauw SA, Lawson JA, van Vlijmen-van Keulen CJ, Pronk P, Gaastra MTW, Mooij MC. Five-year follow-up of a randomized, controlled trial comparing saphenofemoral ligation and stripping of the great saphenous vein with endovenous laser ablation using local tumescent anesthesia. Journal of Vascular Surgery 63(2): 420-428.

8. Bruning G, Schinagl H. Die operative Sanierung des inguinalen Crossenrezidivs mittels modifiziertem Zugang nach Junod. J Dtsch Dermatol Ges. 2011; 9(8): 646-647.

9. Hofer T. Komplikationen nach varizenchirurgischen Eingriffen. Phlebologie 2001; 30: 26-30.

10. Hamann SAS, Giang J, De Maeseneer MGR, Nijsten TEC, van den Bos RR. Editor's Choice Five Year Results of Great Saphenous Vein Treatment: A Meta-analysis. Eur J Vasc Endovasc Surg 2017; 54(6): 760-770.

11. Rass K, Frings N, Glowacki P, Graber S, Tilgen W, Vogt T. Same Site Recurrence is More Frequent After Endovenous Laser Ablation Compared with High Ligation and Stripping of the Great Saphenous Vein: 5 year Results of a Randomized Clinical Trial (RELACS Study). Eur J Vasc Endovasc Surg 2015; 50(5): 648-656. 